\title{
Curativo impregnado com clorexidine para cateter venoso central: análise de teste piloto
}

\author{
Chlorhexidine-impregnated dressing for central venous catheter: pilot clinical trial \\ Curativo impregnado en clorhexidina para catéter venoso central: análisis de prueba piloto
}

\author{
Edivane Pedrolo'; Michelle Caroline Santos ${ }^{\text {II }}$; Gabriella Lemes Rodrigues de Oliveiralit; \\ Priscila Mingorance ${ }^{I V}$; Mitzy Tannia Reichembach Danski ; Radamés Boostel ${ }^{V I}$
}

\begin{abstract}
RESUMO: Objetivou-se realizar teste piloto do ensaio clinico randomizado: avaliação da efetividade do curativo impregnado com clorexidina para cobertura de cateter venoso central. A pesquisa clinica foi desenvolvida nos centros de terapia intensiva e semi-intensiva adulto de um hospital universitário da cidade de Curitiba-PR. A coleta de dados ocorreu entre outubro e novembro de 2011. Os pacientes incluídos foram randomizados para dois grupos: curativo transparente de poliuretano (controle) e curativo antimicrobiano de clorexidina (intervenção). Foram pesquisados oito pacientes. Houve um caso de infecção da corrente sanguínea associada ao cateter e um caso de colonização do cateter, ambos no grupo controle. O curativo transparente necessita de troca antecipada em relação ao tempo estipulado devido à má fixação e ao acúmulo de exsudato sob a película, expondo o paciente a maior risco de colonização e de infecção da corrente sanguínea associada ao cateter. Palavras-Chave: Ensaio clínico; clorexidina; cateterismo venoso central; tecnologia.
\end{abstract}

\begin{abstract}
This randomized pilot clinical trial aimed to evaluate the effectiveness of chlorhexidine-impregnated dressings covering central venous catheters. Data was collected between October and November 2011 at the adult intensive and semiintensive care units of a university hospital in Curitiba, Paraná, Brazil. The eight patients included in the trial were randomized into two groups: transparent polyurethane film dressing (control) and chlorhexidine-impregnated dressing. There was one case of blood stream infection associated with the catheter and one of catheter colonization, both in the control group. The transparent polyurethane film needs to be changed earlier than specified, due to poor fixation and the accumulation of exudate under the film, which can expose patients to greater risk of colonization and infection of the blood stream associated with the catheter. Keywords: Clinical trial; chlorhexidine; central venous catheter; technology.
\end{abstract}

RESUMEN: Este trabajo tuvo por objetivo realizar prueba piloto del ensayo clínico aleatorizado: evaluación de la efectividad del curativo impregnado en clorhexidina para cobertura de catéter venoso central. La investigación clínica se desarrolló en los centros de terapia intensiva y semi-intensiva adulto de un hospital universitario en Curitiba-PR-Brasil. La recogida de datos ocurrió entre octubre y noviembre de 2011. Los pacientes incluidos fueron aleatorizados parados grupos: curativo transparente de poliuretano (control) y curativo antimicrobiano de clorhexidina (intervención). Fueron investigados ocho pacientes. Hubo un caso de infección de la corriente sanguínea asociada al catéter y un caso de colonización del catéter, ambos en el grupo de control. El curativo transparente necesita de cambio anticipado con relación al tiempo estipulado debido a la mala fijación y a la acumulación del exudado bajo la película, exponiendo el paciente a mayor riesgo de colonización y de infección de la corriente sanguínea asociada al catéter.

Palabras Claves: Ensayo clínico; clorhexidina; cateterismo venoso central; tecnología.

\section{INTRODUÇÃO}

O internamento de pacientes em centros de terapia intensiva (CTI) e semi-intensiva (CTSI) requer o emprego de tecnologias avançadas para terapia e cuidado, entre as quais os dispositivos intravenosos, com destaque para o cateter venoso central $(\mathrm{CVC})^{\mathrm{VII}}$. No entanto, o uso de cateteres centrais está relacionado a elevados índices de morbidade e de mortalidade, fato que requer a adoção de medidas de prevenção de complicações, principalmente as complicações infecciosas. Nesse contexto, novas tecnologias têm surgido nos últimos anos com vistas a reduzir as taxas de complicações infecciosas relacionadas a cateteres. Entre

'Enfermeira, Mestre em Enfermagem. Instituto Federal do Paraná. Curitiba, Paraná, Brasil. E-mail: edivane.pedrolo@ifpr.edu.br.

IIAcadêmica de Enfermagem da Universidade Federal do Paraná. Curitiba, Paraná, Brasil. E-mail: chelli_carol@hotmail.com.

IIIEnfermeira. Mestranda em Enfermagem do Programa de Pós-Graduação em Enfermagem da Universidade Federal do Paraná. Curitiba, Paraná, Brasil. E-mail: gabriella.lemes@yahoo.com.br.

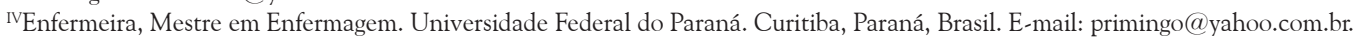

vEnfermeira, Doutora em História. Departamento de Enfermagem da Universidade Federal do Paraná. Curitiba, Paraná, Brasil. E-mail: profa.mitzy@ufpr.br.

VIEnfermeiro, Especialista em Urgência e Emergência. Instituto Federal do Paraná. Curitiba, Paraná, Brasil. E-mail: radames.boostel@ifpr.edu.br.

VII Artigo extraído da dissertação de mestrado intitulada Curativo de clorexidina para cateter venoso central: ensaio clínico randomizado, apresentada ao

Programa de Pós-Graduação em Enfermagem da Universidade Federal do Paraná. 
elas destaca-se o desenvolvimento de curativos específicos para cobertura do óstio do cateter, como o curativo impregnado com clorexidina, uma solução antisséptica amplamente empregada no ambiente hospitalar. No entanto, a adoção desse curativo como material de escolha para cobertura de cateteres centrais requer evidências científicas, as quais podem ser produzidas com a realização de um ensaio clínico randomizado.

Frente ao exposto, a presente pesquisa objetivou realizar o teste piloto de uma pesquisa clínica, desenhada para avaliar a efetividade do curativo impregnado com clorexidina para cobertura de cateter venoso central.

\section{REVISÃO DE LITERATURA}

Os CVC's são dispositivos amplamente utilizados devido aos benefícios que apresentam, entretanto estão relacionados a uma série de complicações, tanto infecciosas quanto não infecciosas. As infecções podem ocorrer durante a inserção e/ou manutenção do cateter ${ }^{1}$.

Entre as complicações infecciosas destaca-se a infecção primária da corrente sanguínea (IPCS), que está comumente relacionada ao uso do CVC, prolongando o tempo de internação dos pacientes e elevando os gastos com seu tratamento. A IPCS corresponde a uma infecção sistêmica grave sem foco primário identificável. Pode estar relacionada à realização de qualquer procedimento invasivo, no entanto considera-se que quando ocorre em pacientes com uso de CVC por mais de 48 horas, está relacionada à presença deste dispositivo. $\mathrm{O}$ diagnóstico IPCS pode ser estabelecido a partir de sinais clínicos inflamatórios (locais e sistêmicos) ou de dados laboratoriais, onde se obtém a confirmação microbiológica da infecção. Destaca-se a relevância dessa complicação, uma vez que cerca de 69\% dos pacientes internados em CTI vão a óbito devido à ocorrência de IPCS ${ }^{2-4}$.

Visando prevenir essa complicação, uma série de cuidados são necessários durante e após a inserção do dispositivo. Devido à solução de continuidade presente no óstio do cateter, o mesmo deve ser protegido com curativo oclusivo estéril ${ }^{4}$. Atualmente, estão disponíveis no mercado curativos dos seguintes materiais: gaze e fita adesiva, filme transparente de poliuretano (FTP) e impregnado com clorexidina ${ }^{1,5}$.

Quando comparado com o curativo de gaze e fita, o FTP apresenta vantagens por seu tempo de permanência maior, redução do custo dos materiais e possibilidade de observação do óstio do cateter sem a necessidade de remoção do curativo ${ }^{5}$. Entre os curativos impregnados, destaca-se o curativo antimicrobiano de clorexidina (CHG), composto por filme transparente associado à clorexidina 2\%, a qual está concentrada em uma placa de gel ou em uma esponja, dependendo do fabricante. Este curativo também possui a vantagem de observação do óstio do cateter sem precisar retirá-lo ${ }^{6}$.
Destaca-se que os curativos de gaze e fita e transparente de poliuretano foram amplamente estudados nos últimos anos, não havendo evidências que indiquem o uso de um em detrimento do outro. No tocante ao curativo impregnado com clorexidina, os estudos são incipientes ${ }^{4,7}$.

\section{Metodologia}

Trata-se do teste piloto de um ensaio clínico randomizado. $\bigcirc$ presente estudo foi aprovado pelo Comitê de Ética em Pesquisa de uma Universidade Federal da cidade de Curitiba-PR, sob registro CEP/ SD 1145.070.11.06 e CAAE 0067.0.091.208-11.

A coleta de dados ocorreu no CTI e CTSI Adulto de um Hospital Universitário da cidade de CuritibaPR, nos meses de outubro e novembro de 2011. A seleção do paciente para a pesquisa esteve sujeita aos seguintes critérios de inclusão: assinatura do Termo de Consentimento Livre e Esclarecido (TCLE); maiores de 18 anos; internação no CTI ou CTSI Adulto; em uso do primeiro CVC de curta permanência há menos de 24 horas; ausência de sensibilidade conhecida aos materiais dos curativos. Os critérios de exclusão foram: submissão à tricotomia por lâmina no sítio de inserção do cateter anteriormente à punção; presença de hemograma infeccioso anteriormente à punção do cateter.

Os pacientes que atenderam aos critérios de inclusão foram selecionados mediante a técnica de randomização em blocos de seis indivíduos ${ }^{8}$, para compor o grupo controle (curativo transparente de poliuretano - FTP) e o grupo de estudo ou de intervenção (curativo antimicrobiano de clorexidina - CHG).

Realizou-se acompanhamento diário e troca dos curativos a cada sete dias nos dois grupos, ou precocemente em caso de comprometimento da integridade do mesmo por descolamento de bordas, presença de exsudato, umidade ou sujidade externa. A coleta de dados e a troca dos curativos foram realizados exclusivamente por equipe capacitada, após padronização do procedimento a ser empregado.

Foram coletadas variáveis sociodemográficas, clínicas e de desfecho, de acordo com um instrumento de coleta de dados previamente elaborado. As variáveis de desfecho analisadas foram: infecção primária da corrente sanguínea, reação local ao curativo e fixação dos curativos. Para análise dos dados utilizou-se estatística descritiva. Os dados foram digitados e tabulados em planilha eletrônica no programa Microsoft Office Excel® 2007.

\section{Resultados e Discussão}

A amostra constituiu-se de oito pacientes, sendo cinco do grupo controle (FTP) e três do grupo estudo/ intervenção $(\mathrm{CHG})$. A maioria é de origem caucasiana e esteve internada no CTI. Do total de pacientes 
incluídos, cinco pertencem ao gênero masculino, com idade média de 58,1 anos, mínima de 37 e máxima de 91 anos, conforme a Tabela 1.

No que concerne às variáveis clínicas, o tempo médio de internação dos pacientes foi de 10,1 \pm 7,2 dias, com mínimo de 2 e máximo de 24. A maioria dos pacientes (cinco) recebeu alta, enquanto o restante (três) foi a óbito.

Para coleta dos diagnósticos, considerou-se o diagnóstico principal no momento da admissão dos pacientes, elencados de acordo com a Classificação Internacional de Doenças (CID-10) ${ }^{9}$. Houve predominância das doenças relacionadas ao aparelho geniturinário (37\%) e ao sistema circulatório (25\%).

Foram encontradas 14 comorbidades entre os sujeitos da pesquisa. Dois pacientes não apresentaram comorbidades, entretanto a maioria teve duas ou mais patologias, fato que justifica o elevado número de comorbidades encontradas. As principais comorbidades existentes foram hipertensão arterial sistêmica (28,6\%) e Diabetes Mellitus (21,4\%), doenças também frequentes em estudo que descreveu o perfil dos pacientes internados em centro de terapia intensiva adulto $(\mathrm{n}=37)^{10}$.

Do total de pacientes analisados, quatro tiveram prescrição de terapia antimicrobiana anteriormente à punção do CVC. Destes, três pacientes utilizaram apenas um antimicrobiano, enquanto um deles utilizou dois. Na literatura não há relato da relação entre utilização de antimicrobiano e aumento do risco de complicações infecciosas.

Quanto às características relacionadas ao cateter, todos eram de duplo lúmen, confeccionados de poliuretano e foram inseridos com a finalidade de infusão de droga vasoativa. Pesquisadores afirmam que cada lúmen aumenta de 15 a 20 vezes/dia à manipulação do cateter, ampliando assim os riscos de infecção ${ }^{11}$. Em estudo epidemiológico realizado com 630 pacientes, no qual foram incluídos cateteres mono, duplo e triplo lúmen, constatou-se a ocorrência de 40 casos de IPCS, das quais $85 \%$ relacionadas à utilização de cateteres duplo lúmen ${ }^{12}$.

A média geral de permanência do CVC foi de 5,1 dias, com mediana de 5,5, mínimo de 1 e máximo de

TABELA 1: Características dos pacientes e dos cateteres nos grupos pesquisados. Curitiba, PR, 2012.

\begin{tabular}{|c|c|c|c|c|}
\hline \multirow[t]{3}{*}{ Características } & \multicolumn{4}{|c|}{ Grupo } \\
\hline & \multicolumn{2}{|c|}{$\begin{array}{l}\text { Filme transparente de } \\
\text { poliuretano } \\
(\mathrm{n}=5)\end{array}$} & \multicolumn{2}{|c|}{$\begin{array}{c}\text { Curativo } \\
\text { antimicrobiano de } \\
\text { clorexidina } \\
(n=3)\end{array}$} \\
\hline & $f$ & $\%$ & $f$ & $\%$ \\
\hline \multicolumn{5}{|l|}{ Sexo } \\
\hline Feminino & 1 & 20 & 2 & 66,6 \\
\hline Masculino & 4 & 80 & 1 & 33,3 \\
\hline \multicolumn{5}{|l|}{ Idade } \\
\hline Média & 60,6 & & 54 & \\
\hline \multicolumn{5}{|l|}{ Procedência } \\
\hline Centro de terapia intensiva & 3 & 60 & 3 & 100 \\
\hline Centro de terapia semi-intensiva & 2 & 40 & 0 & 0 \\
\hline \multicolumn{5}{|l|}{ Indicação do CVC } \\
\hline Infusão de droga vasoativa & 5 & 100 & 3 & 100 \\
\hline \multicolumn{5}{|l|}{ Setor de punção } \\
\hline Centro Cirúrgico & 4 & 80 & 2 & 66,6 \\
\hline Centro de terapia semi-intensiva & 1 & 20 & 1 & 33,3 \\
\hline \multicolumn{5}{|l|}{ Sítio de inserção } \\
\hline Subclávia direita & 4 & 80 & 2 & 66,6 \\
\hline Jugular esquerda & 1 & 20 & 1 & 33,3 \\
\hline \multicolumn{5}{|l|}{ Tempo de permanência do cateter } \\
\hline Média \pm Desvio padrão & $7 \pm 2,9$ & & 2 & \\
\hline \multicolumn{5}{|l|}{ Motivo de retirada } \\
\hline Eletiva & 3 & 60 & 2 & 66,6 \\
\hline Suspeita de infecção & 1 & 20 & 0 & 0 \\
\hline Óbito & 1 & 20 & 1 & 33,3 \\
\hline
\end{tabular}


10 dias. A permanência do cateter por tempo superior a 5 dias é indicada por autores como fator de risco para o desenvolvimento de IPCS ${ }^{13}$. A presente pesquisa apresentou um caso de IPCS, sendo este identificado em um sujeito com uso de CVC por 7 dias.

No tocante ao setor de punção do cateter, seis foram puncionados no centro cirúrgico (CC) e dois no CTSI; os sítios de inserção correspondem à veia subclávia direita e jugular esquerda, em seis e dois sujeitos respectivamente. Observou-se preferência pela inserção do cateter nas veias subclávia direita e jugular esquerda, conforme demonstrado na Tabela 1 , contudo não há evidências seguras relacionando os dois sítios com a presença de infecção ${ }^{14}$. A recomendação de maior nível de evidência científica orienta a inserção de cateteres de curta permanência, preferencialmente, em veia subclávia, bem como evitar a punção da veia femural para este tipo de dispositivo ${ }^{4}$.

Quanto ao motivo de retirada do cateter, um paciente teve sua retirada por suspeita de infecção (não confirmada), dois foram a óbito e cinco tiveram retirada eletiva do cateter, ou seja, o dispositivo não era mais necessário, segundo a Tabela 1 .

O tempo de permanência dos dois tipos de curativo foi semelhante, com média de 2,5 dias para o filme transparente e de 2 dias para o curativo de $\mathrm{CHG}$. Destaca-se que apenas o curativo filme transparente de poliuretano necessitou de troca antecipada, das quais seis foram devidas à má fixação e duas por exsudato abundante.

Para as variáveis relacionadas à infecção (febre, hipotensão, oligúria, hiperemia, cianose e exsudato no óstio do cateter, enduração, dor ou edema à palpação) e reação local (prurido, hiperemia, descamação e maceração), foram contabilizados os pacientes que apresentaram estes sinais em pelo menos 70\% dos dias de acompanhamento. Somente duas variáveis foram identificadas, em apenas um paciente: presença de hiperemia na pele e no óstio de inserção. Este dado diverge de ensaio clínico randomizado realizado com 21 pacientes, que avaliou o curativo transparente como cobertura para CVC, no qual a hiperemia foi o terceiro sinal mais frequente, observado em $33 \%$ dos pacientes?

Quanto à capacidade de fixação do filme transparente, esta foi considerada boa em 15 dias, ruim em 7 dias e presença de borda descolada em 11 dias, totalizando 33 dias de acompanhamento. $\mathrm{O}$ curativo transparente de poliuretano necessitou de troca antecipada, em relação às recomendações internacionais ${ }^{4}$, devido à presença de exsudato (duas) e à má fixação (cinco). Já para o curativo de $\mathrm{CHG}$, a fixação foi considerada boa em todos os dias de acompanhamento.

O tempo de permanência médio do curativo de CHG foi de 2 dias, enquanto do curativo transparente foi de 2,5 dias. Os resultados vão ao encontro de outro estudo $(n=21)$, que apresenta permanência média de
1,9 dias para o curativo transparente ${ }^{7}$. Destaca-se que o tempo de permanência do curativo de clorexidina é de até 7 dias, entretanto, na presente pesquisa este foi inferior devido ao curto tempo de permanência do cateter no grupo de pacientes em uso dessa tecnologia, que foi de 2 dias em média.

Houve um caso de infecção da corrente sanguínea associada ao cateter e um caso de colonização do cateter, com isolamento do microorganismo Staphylococcus coagulase-negativo. Esses dados vão ao encontro de estudos que trazem as bactérias Gram positivas como principais agentes etiológicos de IPCS, sendo Staphylococcus coagulase-negativo o mais frequente $\mathrm{e}^{4,15,16}$.

Da totalidade de oito pacientes, três utilizaram o curativo antimicrobiano de clorexidina. Metanálise demonstrou uma redução de $60 \%$ de infecções associadas ao CVC quando utilizado este curativo ${ }^{17}$. Ademais, exames de culturas de pele de pacientes em uso de curativos de $\mathrm{CHG}$ demonstram significativa redução de presença de microorganismos com potencial patogênico em relação ao curativo transparente convencional.

$\mathrm{Na}$ literatura nacional ainda não existem estudos em relação ao curativo antimicrobiano de clorexidina. Já, na literatura internacional, ensaio clínico randomizado comparou este curativo com o filme transparente de poliuretano, constatando uma redução dos casos de IPCS com o uso do curativo de $\mathrm{CHG}^{18}$.

A inserção de tecnologias e de inovações no campo da enfermagem é uma necessidade e uma realidade. Entretanto, apesar das tecnologias trazerem uma série de vantagens para o trabalho da enfermagem, entre elas maior segurança no cuidado ${ }^{19}$, há necessidade de avaliar sua efetividade. Dessa forma, a presente pesquisa contribui para a incorporação de uma inovação tecnológica para o cuidado com o cateter venoso central, com vistas a reduzir os índices de complicações infecciosas relacionadas a esse dispositivo, as quais oneram tanto os serviços quanto a condição de saúde dos pacientes.

\section{Conclusão}

Destaca-se que o tipo de curativo utilizado para a cobertura do cateter interferiu nos resultados finais. Os desfechos de infecção primária da corrente sanguínea e de colonização do cateter ocorreram em pacientes que fizeram uso do curativo transparente. Quanto à capacidade de fixação, indicou-se que o resultado com o curativo de CHG é mais satisfatório, visto que o curativo transparente necessita de troca antecipada em relação ao tempo estipulado, devido à má fixação e ao acúmulo de exsudato sob a película, expondo o paciente a um maior risco de colonização e de infecção da corrente sanguínea associada ao cateter. 
Ressalta-se como limitações da pesquisa a pequena amostra, que impossibilita a generalização dos achados. Entretanto, cumpriu com os propósitos de um teste piloto e os dados referentes à colonização e a IPCS foram fundamentais para reformulação do protocolo de pesquisa clínica.

\section{REFERÊNCIAS}

1.Harada MJCS, Pedreira MLG, organizadoras. Terapia intravenosa e infusões. São Caetano do Sul (SP): Yendis; 2011. 2.Ministério da Saúde (Br). Agência Nacional de Vigilância Sanitária. Unidade de Investigação e Prevenção das Infecções e dos Efeitos Adversos - UIPEA. Corrente sanguínea: critérios nacionais de infecção relacionadas à assistência à saúde. Brasília (DF): Ministério da Saúde; 2009. 3.Ministério da Saúde (Br). Agência Nacional de Vigilância Sanitária. Unidade de Investigação e Prevenção das Infecções e dos Efeitos Adversos - UIPEA. Orientações para prevenção de infecção primária de corrente sanguínea. Brasília (DF): Ministério da Saúde; 2010. 4.O'Grady NP, Alexander M, Burns LA, Dellinger P, Garland J, Heard SO, et al. Guidelines for the prevention of intravascular catheter-related infections. Centers of disease control and prevention (CDC) 2011 [cited in 2014 Sep 15]. 52(9):1-83. Available in: http://www.cdc. gov/hicpac/pdf/guidelines/bsi-guidelines-2011.pdf

5.Silveira R, Braga FTMM, Garbin LM, Galvão CM. O uso do filme transparente de poliuretano no cateter venoso central de longa permanência. Rev Latino-Am Enferm. 2010 [citado em 15 set 2014]. 18(6). Disponível em: http://www.scielo.br/pdf/rlae/v18n6/pt_23.pdf

6.Oliveira GLR. Perfil epidemiológico dos pacientes em uso do cateter venoso central em um centro de terapia intensiva [monografia]. Curitiba (PR): Universidade Federal do Paraná; 2012.

7.Pedrolo E, Danski MTR, Mingorance P, De Lazzari LSM, Johann DA. Clinical controlled trial on central venous catheter dressings. Acta Paul Enferm. 2011 [cited in 2014 Sep 15]. 24:278-83. Available in: http://www. scielo.br/pdf/ape/v24n2/19.pdf

8.Hulley SB, Cumming SR, Browner WS, Grady DG, Newman TB. Delineando a pesquisa clínica: uma abordagem epidemiológica. $3^{a}$ ed. Porto Alegre (RS): Artmed; 2008.

9.Organização Mundial da Saúde. Classificação Estatística Internacional de Doenças e Problemas Relacionados à Saúde (CID-10). 10 rev. São Paulo: Universidade de São Paulo; 2008 [citado em 11 jun 2012]. Disponível em: http:// www.datasus.gov.br/cid10/v2008/webhelp/cid10.htm. 10.Marques Netto S, Echer IC, Kuplich NM, Kuchembecker R, Kessler F. Infecção de cateter vascular central em pacientes adultos de um centro de terapia intensiva. Rev Gaúcha Enferm. 2009 [citado em 15 set 2014].
30: 429-36. Disponível em: http://seer.ufrgs.br/RevistaGauchadeEnfermagem/article/view/8957/6964.

11.Associação Paulista de Estudos e Controle de Infecção Hospitalar. Infecção relacionada ao uso de cateteres vasculares. São Paulo: APECIH; 2005.

12.Mesiano ERAB, Merchán-hamann M. Infecções da corrente sanguínea em pacientes em uso de cateter venoso central em unidades de terapia intensiva. Rev Latino-Am Enferm. 2007 [citado em 15 set 2014]. 15(3). Disponível em: http://www.scielo.br/pdf/rlae/v15n3/pt_v15n3a14.pdf. 13.Porto JP, Dantas RCC, Freitas C, Matoso DC, Almeida $\mathrm{AB}$, Gontijo Filho PP, et al. Bloodstream infection associated/related to the central venous catheter in mixed ICU of adults from a Brazilian university hospital: etiology, pathogenesis and risk factors. Rev Panam Infectol. 2010 [cited in 2014 Sep 15]. 12(2):24-9. Available in: http:// www.revista-api.com/2010/pdf/02/API_02_10_D.pdf. 14.Siqueira GLG, Hueb W, Contreira R, Nogueron MA, Cancio DM, Caffaro RA. Infecção de corrente sanguínea relacionada a cateter venoso central (ICSRC) em enfermarias: estudo prospectivo comparativo entre veia subclávia e veia jugular interna. Rev Vasc Bras. 2011 [citado em 15 set 2014]. 10(3): 211-6. Disponível em: http://www.scielo.br/pdf/jvb/v10n3/05.pdf.

15.Ross C, Quesada RMB, Girardello R, Rogeri LMS, Calixto LA, Pelayo JS. Análise microbiológica de pontas de catéteres venosos centrais provenientes de pacientes internados no Hospital Universitário da Universidade Estadual de Londrina. Semina. 2006 [citado em 15 set 2014]. 27(2):117-23. Disponível em: http://www.uel.br/ revistas/uel/index.php/seminabio/article/view/3506/2843. 16.Bernardi ACA, Pizzolitto EL, Pizzolitto AC. Detecção da produção de slime por estafilococos coagulase-negativa isolados de cateter venoso central. Rev Ciênc Farm Básica Apl. 2007 [citado em 15 set 2014]. 28(1):57-66. Disponível em: http://200.145.71.150/seer/index.php/ Cien_Farm/article/viewFile/346/331.

17.Ho KM, Litton E. Use of chlorhexidine-impregnated dressing to prevent vascular and epidural catheter colonization and infection: a meta-analysis. J Antimicrob Chemoth. 2006 [cited in 2014 Sep 15]. 58(2):281-7. Disponível em: http://jac.oxfordjournals.org/content/58/2/281.full.pdf + html.

18. Timsit JF, Schwebel C, Bouadma L, Geffroy A,Garrouste-Orgeas M, Pease S, et al. Chlorhexidineimpregnated sponges andlessfrequentdressingchangesforprevention ofcatheter-relatedinfections incritically ill adults: a randomized controlled trial. JAMA. 2009 [cited in 2014 Sep 15]. 301: 1231-41. Available in: http://jama. jamanetwork.com/article.aspx?articleid = 183597 .

19.Salvador PTCO, Oliveira RKM, Costa TD, Santos VEP, Tourinho FSV. Tecnologia e inovação para o cuidado em enfermagem. Rev enferm UERJ. 2012 [citado em 15 set 2014]. 20:111-7. Available in: http://www.facenf.uerj. br/v20n1/v20n1a19.pdf. 